\title{
Log intermediate Jacobians
}

\author{
By Kazuya Kato, $\left.{ }^{*}\right)$ Chikara Nakayama, ${ }^{* *}$ and Sampei Usui***) \\ (Communicated by Masaki KashiwARA, M.J.A., March 12, 2010)
}

\begin{abstract}
We study the degenerations of intermediate Jacobians by means of log geometry. We extend the family of intermediate Jacobians over a punctured disc to a "log intermediate Jacobian" over a disc.

Key words: Hodge theory; log geometry; intermediate Jacobian; Néron model; admissible normal function; log intermediate Jacobian.
\end{abstract}

Introduction. Let $S=\Delta=\{q \in \mathbf{C}|| q \mid<1\}$, and let $f: X \rightarrow S$ be a projective morphism which is smooth over $S^{*}=\Delta^{*}=\Delta \backslash\{0\}$ and with semistable reduction at $q=0$.

Put $X^{*}=f^{-1}\left(S^{*}\right)$. Fix a polarization of $X / S$ and fix $r \geq 1$. Then we have the variation of polarized Hodge structure $H^{2 r-1}\left(X^{*} / S^{*}\right)$ over $S^{*}$ underlain by $R^{2 r-1} f_{*} \mathbf{Z} /$ (torsion) (cf. [Gri70]). Twist it into $H^{2 r-1}\left(X^{*} / S^{*}\right)(r)$ whose weight is -1 . Fix a point $s \in S^{*}$.

Define Z-modules $H^{\prime}$ and $H$ as $H^{\prime}:=$ $H_{\mathbf{Z}}^{2 r-1}\left(X^{*} / S^{*}\right)(r)_{s}, H:=H^{\prime} \oplus \mathbf{Z}$. Let $W$ be the increasing filtration on $H_{\mathbf{Q}}$ defined by $W_{-2}=0$, $W_{-1}=H_{\mathbf{Q}}^{\prime}, \quad W_{0}=H_{\mathbf{Q}}$. Let $\langle,\rangle_{0}: \mathbf{Z} \times \mathbf{Z} \rightarrow \mathbf{Q}$; $(a, b) \mapsto a b$, and $\langle,\rangle^{\prime}=\langle,\rangle_{-1}: H^{\prime} \times H^{\prime} \rightarrow \mathbf{Q}$ be the pairing defined by the polarization. Let $\left(h_{k}^{p, q}\right)$ be the evident Hodge numbers.

Let $D^{\prime}=D\left(H^{\prime},\left(h_{-1}^{p, q}\right),\langle,\rangle^{\prime}\right)$ be the classifying space of polarized Hodge structures of weight -1 in [Gri68], and let $D=D\left(H, W,\left(h_{k}^{p, q}\right),\left(\langle,\rangle_{k}\right)\right)$ be the classifying space of gradedly polarized mixed Hodge structures in [Usu84]. Then $D^{\prime}=\operatorname{gr}_{-1}^{W}(D)$. Let $\gamma^{\prime}: H^{\prime} \rightarrow H^{\prime}$ be the local monodromy, i.e., the action of the standard generator of $\pi_{1}\left(S^{*}\right)$, and let $\Gamma^{\prime} \subset \operatorname{Aut}\left(H^{\prime},\langle,\rangle^{\prime}\right)$ be the group generated by $\gamma^{\prime}$. Let $\Gamma$ be the subgroup of $G_{\mathbf{Z}}=\operatorname{Aut}\left(H, W,\left(\langle,\rangle_{k}\right)\right)$ consisting of all elements whose restrictions to $H^{\prime}$ are contained in $\Gamma^{\prime}$ and which induce 1 on $\operatorname{gr}_{0}^{W}(H)=\mathbf{Z}$.

2000 Mathematics Subject Classification. Primary 14C30; Secondary $14 \mathrm{D} 07,32 \mathrm{G} 20$.

*) Department of Mathematics, University of Chicago, $5734 \mathrm{~S}$. University Avenue, Chicago, Illinois 60637, USA.

**) Graduate School of Science and Engineering, Tokyo Institute of Technology, Meguro-ku, Tokyo 152-8551, Japan.

***) Graduate School of Science, Osaka University, Toyonaka, Osaka 560-0043, Japan.
Let $J_{X^{*} / S^{*}}^{r}:=\left(H_{\mathcal{O}}^{2 r-1}\left(X^{*} / S^{*}\right) /\left(F^{r}+H_{\mathbf{Z}}^{2 r-1}\left(X^{*} /\right.\right.\right.$ $\left.\left.\left.S^{*}\right)\right)\right)(r)$ be the intermediate Jacobian introduced by Griffiths (cf. [GS69]).

Then there is an isomorphism of functors (cf. [Car80])

$$
\operatorname{Mor}\left(?, J_{X^{*} / S^{*}}^{r}\right) \simeq \mathcal{E} x t_{M H S}^{1}\left(\mathbf{Z}, H^{2 r-1}\left(X^{*} / S^{*}\right)(r)\right)
$$

from the category of complex spaces over $S^{*}$ to the category of sets. Here $\mathcal{E} x t^{1}$ is taken in the category (MHS) of mixed Hodge structures with polarized graded quotients. This is also expressed as the fiber product (cf. [Usu84])

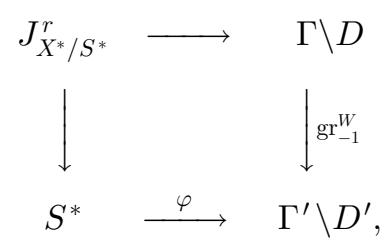

where $\varphi$ is the period map associated to the variation of polarized Hodge structure $H^{2 r-1}\left(X^{*} / S^{*}\right)(r)$ in [Gri68].

The purpose of this article is to extend $J_{X^{*} / S^{*}}^{r}$ to a "log intermediate Jacobian $J_{X / S, \Sigma}^{r}$ " over $S$ by using log geometry ( $\S 4)$. Our " $J_{X / S, \Sigma}^{r}$ " is related to works by Zucker [Zuc76], Clemens [Cle83], and Saito [Sai96] (§5).

$\S \S 1-7$ in this article are based on our note on which the third author gave a talk at the JAMI conference at Johns Hopkins university in March, 2005.

We omit the details of constructions and proofs here, which are to be published elsewhere.

1. Associated log Hodge structure. Endow $X$ and $S$ with the standard log structures defined by $f^{-1}(0)$ and $\{0\}$ respectively ([Kak89, Ill94]).

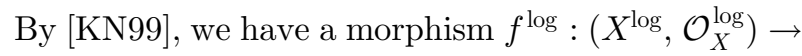
$\left(S^{\log }, \mathcal{O}_{S}^{\log }\right)$ of ringed spaces. [Usu01] showed that 
$f^{\log }: X^{\log } \rightarrow S^{\log }$ is topologically locally trivial over the base.

We have the polarized log Hodge structure $H^{2 r-1}(X / S)$ over $S$ of weight $2 r-1$ underlain by $R^{2 r-1} f_{*}^{\log } \mathbf{Z} /$ (torsion) ([Kaf98, Mat98, Fuj99, KU99, KMN02, KU09]). (Recall that a polarized log Hodge structure of weight $k$ over $S$ is a triple $\left(L_{\mathbf{Z}},\langle\rangle, F,\right)$, where $L_{\mathbf{Z}}$ is a locally constant sheaf of finitely generated free $\mathbf{Z}$-modules on $S^{\log },\langle$,$\rangle is a (-1)^{k}$-symmetric bilinear form $L_{\mathbf{Z}} \times L_{\mathbf{Z}} \rightarrow \mathbf{Q}$, and $F$ is a decreasing filtration of the $\mathcal{O}_{S}^{\log }$-module $\mathcal{O}_{S}^{\log } \otimes_{\mathbf{Z}} L_{\mathbf{Z}}$, satisfying some conditions. See [KU99] $\S 2$ for details.) This is a reformulation, in terms of log Hodge theory, of the classical theory of Schmid [Sch73], and Steenbrink [Ste76] which says that a limit Hodge structure appears at $q=0$.

Consider its twist $H^{2 r-1}(X / S)(r)$ of weight -1 .

2. Main theorem. Let $N^{\prime}=\log \left(\gamma^{\prime}\right): H_{\mathrm{Q}}^{\prime} \rightarrow$ $H_{\mathbf{Q}}^{\prime}$ (note that $\gamma^{\prime}$ is unipotent). Let $\Sigma^{\prime}$ be the fan in $\mathfrak{g}^{\prime}:=\operatorname{End}\left(H_{\mathbf{R}}^{\prime},\langle,\rangle^{\prime}\right)=\left\{h: H_{\mathbf{R}}^{\prime} \rightarrow H_{\mathbf{R}}^{\prime} \mid\langle h(x), y\rangle^{\prime}+\right.$ $\left.\langle x, h(y)\rangle^{\prime}=0\right\}$ defined by $\Sigma^{\prime}=\left\{\left(\mathbf{R}_{\geq 0}\right) N^{\prime},\{0\}\right\}$.

Theorem. There is a fan $\Sigma$ in $\mathfrak{g}:=$ $\operatorname{End}\left(H_{\mathbf{R}}, W,\left(\langle,\rangle_{k}\right)\right)$, consisting of rational nilpotent cones, which satisfies the following (1) and (2).

(1) For any $\sigma \in \Sigma, \sigma$ is admissible for $W$ and the restriction of any element of $\sigma$ to $H_{\mathbf{R}}^{\prime}$ is contained in $\left(\mathbf{R}_{>0}\right) N^{\prime}$. Furthermore, $\Sigma$ is strongly compatible with $\Gamma$.

(2) (Relative completeness.) Let $\sigma$ be any rational nilpotent cone in $\mathfrak{g}$ which is admissible for $W$ such that the restriction of any element of $\sigma$ to $H_{\mathbf{R}}^{\prime}$ is contained in $\left(\mathbf{R}_{>0}\right) N^{\prime}$. Then there exists a finite subdivision $\left\{\sigma_{j}\right\}$ of $\sigma$ such that each $\sigma_{j}$ is contained in some element of $\Sigma$.

We explain some terminology in the above theorem. A rational nilpotent cone $\sigma$ in $\mathfrak{g}$ is a cone over $\mathbf{R}_{>0}$ in $\mathfrak{g}$ generated by a finite number of mutually commutative nilpotent elements of $\mathfrak{g}_{\mathbf{Q}}$. We also assume that $\sigma$ is sharp, i.e., $\sigma \cap(-\sigma)=\{0\}$. A nilpotent cone $\sigma$ is admissible for $W$ if, for any element $N$ of $\sigma$, there exists the $W$-relative $N$-filtration $M=M(N, W)$, and furthermore, this filtration $M$ depends only on the smallest face of $\sigma$ containing $N$. See [SZ85, Kas85, Kas86] for the details on admissibility. See [KU09] for the definition of the strong compatibility.

Definition. We call a fan $\Sigma$ in $g$ satisfying the conditions (1) and (2) of Theorem as a relatively complete fan.
3. Example of a relatively complete fan. We will explain the proof of the main theorem by giving an explicit example of a relatively complete fan. Let

$$
\begin{aligned}
& P:=\operatorname{Im}\left(N^{\prime}: H_{\mathbf{Q}}^{\prime} \rightarrow H_{\mathbf{Q}}^{\prime}\right), \\
& Q:=\operatorname{Ker}\left(N^{\prime}: H_{\mathbf{Q}}^{\prime} \rightarrow H_{\mathbf{Q}}^{\prime}\right) \cap P .
\end{aligned}
$$

Take a finitely generated $\mathbf{Z}$-submodule $L$ of $H_{\mathbf{Q}}^{\prime}$ containing $H^{\prime}+N^{\prime}\left(H^{\prime}\right)$. Take a section $s:(P \cap L) /$ $(Q \cap L) \rightarrow P \cap L$ of $\mathbf{Z}$-modules to the surjection $P \cap L \rightarrow(P \cap L) /(Q \cap L)$. Write as $s$ also for the induced $\mathbf{Q}$-linear map $P / Q \rightarrow P$ by abuse of notation. For an element $x$ of $P / Q$, let $a(x)$ be the order of the image of $x$ in $P /((P \cap L)+Q)$.

Fix a Z-basis $\left(e_{j}\right)_{1 \leq j \leq m}$ of $Q \cap L$, and fix an element $e \in H$ inducing $1 \in \mathbf{Z}=\operatorname{gr}_{0}^{W}(H)$.

For $x \in P / Q$ and $n=\left(n_{j}\right)_{j} \in \mathbf{Z}^{m}$, let $\sigma(x, n)$ be the $\left(\mathbf{R}_{\geq 0}\right)$-cone in $\mathfrak{g}$ generated by all elements $N \in \mathfrak{g}$ having the following property:

The restriction of $N$ to $H_{\mathbf{R}}^{\prime}$ coincides with $N^{\prime}$, and $N(e)$ is an element of $H_{\mathbf{R}}^{\prime}$ of the form $s(x)+(1 / a(x)) \sum_{1 \leq j \leq m} c_{j} e_{j}$ with $n_{j} \leq c_{j} \leq n_{j}+1$ for all $j$.

Let $\Sigma$ be the set of all faces of $\sigma(x, n)$ for all $x \in P / Q$ and $n \in \mathbf{Z}^{m}$.

It is easy to see that this $\Sigma$ satisfies the desired conditions (1) and (2).

4. Log intermediate Jacobian. Let $\Sigma$ be a fan satisfying (1) in Theorem.

Let $D_{\Sigma^{\prime}}^{\prime}=D_{\Sigma^{\prime}}\left(H^{\prime},\left(h_{-1}^{p, q}\right),\langle,\rangle^{\prime}\right)$ be the space of nilpotent orbits, and let $D_{\Sigma}=D_{\Sigma}\left(H, W,\left(h_{k}^{p, q}\right)\right.$, $\left.\left(\langle,\rangle_{k}\right)\right)$ be the space of mixed nilpotent orbits. Then $D_{\Sigma^{\prime}}^{\prime}=\operatorname{gr}_{-1}^{W}\left(D_{\Sigma}\right)$. See [KU99, KU09, KNU08, KNU09, KNU.p] for the details.

Proposition. The space $\Gamma \backslash D_{\Sigma}$ is a log manifold and Hausdorff.

This is a main result of [KNU.p], which is a mixed version of [KU09], and the proof is similar to the pure case in loc. cit. As mentioned in [KNU08] 0.8 , in the proof, the $\mathrm{SL}(2)$-orbit theorem 0.5 in loc. cit. plays a key role, that is, to guarantee the continuity of the CKS-map (cf. [KU09]), exactly as the SL(2)-orbit theorem of Cattani-Kaplan-Schmid [CKS86] did so in the pure case [KU09].

The polarized log Hodge structure $H^{2 r-1}(X / S)$ $(r)$ defines the extended period map $S \rightarrow \Gamma^{\prime} \backslash D_{\Sigma^{\prime}}^{\prime}$ by [KU09].

Define $J_{X / S, \Sigma}^{r}$ to be the fiber product 


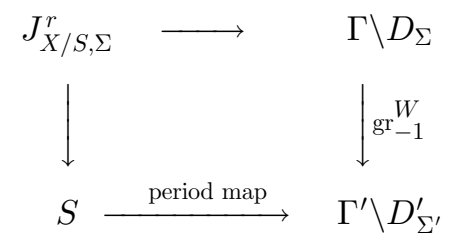

in the category $\mathcal{B}(\log )([\mathrm{KU} 09] 3.2 .4)$.

Proposition. The space $J_{X / S, \Sigma}^{r}$ is a log manifold and Hausdorff.

If a fan $\Upsilon$ also satisfies (1) in Theorem and if $\Upsilon \subset \Sigma$, then $J_{X / S, \Upsilon}^{r}$ is an open subset of $J_{X / S, \Sigma}^{r}$.

For $\Sigma$ which also satisfies (2) in Theorem, i.e., for a relatively complete fan $\Sigma$, we call $J_{X / S, \Sigma}^{r}$ the $r$-th $\log$ intermediate Jacobian associated to $X / S$ and $\Sigma$.

5. Relationship with other works. What we have just constructed is closely related to some works of Zucker [Zuc76] (cf. also [EZ84]), Clemens [Cle83], and Saito [Sai96]. What they considered are essentially $J_{X / S, \Sigma}^{r}$ for the following fans $\Sigma=\Sigma_{j}$ $(j=0,1,2)$. We denote Zucker's (resp. Clemens', Saito's) fan by $\Sigma_{0}$ (resp. $\left.\Sigma_{1}, \Sigma_{2}\right)$. We also denote our relatively complete fan in $\S 3$ by $\Sigma_{3}$. As in [Cle83] and [Sai96], we consider $\Sigma_{1}$ and $\Sigma_{2}$ under the condition:

$$
\left(N^{\prime}\right)^{2}=0 \text { and } \operatorname{gr}_{0}^{W^{\prime}} \text { has Hodge type }(0,0) .
$$

Here $W^{\prime}=W\left(N^{\prime}\right)[1]$, that is, the $N^{\prime}$-filtration on $H^{\prime}$.

Now we define $\Sigma_{j}(j=0,1,2)$ :

$$
\begin{aligned}
\Sigma_{0}= & \left\{\left(\mathbf{R}_{\geq 0}\right) N\left|N \in \mathfrak{g}_{\mathbf{Q}}, N\right|_{H^{\prime}}=N^{\prime}, N(e) \in\right. \\
& \left.N^{\prime}\left(H^{\prime}\right)\right\} \cup\{\{0\}\} . \\
\Sigma_{1}= & \left\{\left(\mathbf{R}_{\geq 0}\right) N\left|N \in \mathfrak{g}_{\mathbf{Q}}, N\right|_{H^{\prime}}=N^{\prime}, N(e) \in\right. \\
& \left.Q \cap H^{\prime}\right\} \cup\{\{0\}\} . \\
\Sigma_{2}= & \text { face of } \left.\sigma_{n} \mid n \in \mathbf{Z}^{m}\right\},
\end{aligned}
$$

where for a fixed $\mathbf{Z}$-basis $e_{1}^{\prime}, \ldots, e_{m}^{\prime}$ of $N^{\prime}\left(H^{\prime}\right), \sigma_{n}$ is the $\left(\mathbf{R}_{\geq 0}\right)$-cone generated by

$$
\begin{array}{r}
\left\{N \in \mathfrak{g}_{\mathbf{Q}}|N|_{H^{\prime}}=N^{\prime}, N(e)=\sum_{1 \leq j \leq m} c_{j} e_{j}^{\prime}\right. \\
\text { with } \left.n_{j} \leq c_{j} \leq n_{j}+1 \text { for any } j\right\} .
\end{array}
$$

Then $\Sigma_{j}$ 's satisfy (1) in Theorem. Further, $\Sigma_{2}$ satisfies also (2) in Theorem.

Under the condition (5.1), we see that $P=Q$ so that $s$ must be 0 , and for any $L$ and some bases of $N^{\prime}\left(H^{\prime}\right)$ and $Q \cap L$, we have the following relationship among the four fans:

$$
\begin{array}{ccc}
\Sigma_{2} & <\Sigma_{3} \\
\cup & & \cup \\
\Sigma_{0} & \subset \Sigma_{1}
\end{array}
$$

$\Sigma_{1}$ and $\Sigma_{2}$ are not necessarily contained in each other. Here $A<B$ means $B$ is a subdivision of $A$.

Precisely speaking, as Saito pointed out in [Sai96, (3.5) (iv)], Zucker's space is not Hausdorff. This is because Zucker did not impose Griffiths transversality. On the other hand, our space $J_{X / S, \Sigma_{0}}^{r}$ or $J_{X / S, \Sigma_{3}}^{r}$ is always Hausdorff thanks to slits coming from Griffiths transversality (see the next section). In the case (5.1), Griffiths transversality is automatically satisfied and hence slits do not appear, and Clemens and Saito considered exactly $J_{X / S, \Sigma_{j}}^{r}$ ( $j=1,2$, respectively) which is Hausdorff. Clemens' is "Néron model" which is not necessarily proper over $S$, whereas Saito's is proper over $S$.

Proposition. Let $\Sigma$ be a fan satisfying (1) in Theorem. Assume (5.1). Then, $J_{X / S, \Sigma}^{r} \rightarrow S$ is proper if and only if $\Sigma$ is relatively complete.

This is due to the fact that slits do not appear under (5.1).

6. Example of slit. Let $Y=\mathbf{C} / \mathbf{Z}[i]$ be the elliptic curve with period $i$, let $g: E \rightarrow \Delta$ be the standard degeneration of elliptic curves, i.e., $g^{-1}(q)=$ $\mathbf{C}^{\times} / q^{\mathbf{Z}}$ for $q \neq 0$ and $g^{-1}(0)$ is a rational curve with one node, and consider the family $f:=g \circ \mathrm{pr}_{2}$ : $X=Y^{2} \times E \rightarrow S=\Delta$. Then we see (Zucker's space for $\left.\Sigma_{0}\right)=Y^{4} \times\left(\left(\left(\mathbf{C}^{\times}\right)^{4} \times \mathbf{C}^{2} \times \Delta\right) / \sim\right)$. Here $\left(\left(t_{j}\right)\right.$, $\left.\left(a_{k}\right), q\right) \sim\left(\left(t_{j}^{\prime}\right),\left(a_{k}^{\prime}\right), q^{\prime}\right)$ if and only if

$$
\left\{\begin{array}{l}
\text { when } q \neq 0 ; \quad q^{\prime}=q, t_{j}^{\prime} / t_{j} \in q^{\mathbf{Z}} \text { for any } j \text {, there } \\
\quad \text { exists } b \in \mathbf{Z}[i] \text { such that } a_{2}^{\prime}-a_{2}-b=0 \\
\quad \text { and } a_{1}^{\prime}-a_{1}-b \cdot \log (q) /(2 \pi i) \in \mathbf{Z}[i] ; \\
\text { when } q=0 ; \quad q^{\prime}=q=0, t_{j}^{\prime}=t_{j} \text { for any } j, \\
\qquad a_{2}^{\prime}-a_{2}=0, a_{1}^{\prime}-a_{1} \in \mathbf{Z}[i] .
\end{array}\right.
$$

This is not Hausdorff. In fact, let $c \in \mathbf{C}$ and $t \in\left(\mathbf{C}^{\times}\right)^{4}$, and for $n \gg 0$, let $q_{n}=\exp (2 \pi i(c+n i))$. Then $\left(t,(c, 1), q_{n}\right) \sim\left(t,(0,0), q_{n}\right)$, and $\left(t,(c, 1), q_{n}\right)$ (resp. $\left.\left(t,(0,0), q_{n}\right)\right)$ converges to $(t,(c, 1), 0)$ (resp. $(t,(0,0), 0))$ as $n \rightarrow \infty$. But we see $(t,(c, 1), 0) \chi$ $(t,(0,0), 0)$.

On the other hand, our space is $J_{X / S, \Sigma_{0}}^{2}=$ $Y^{4} \times\left\{\right.$ class of $\left.(t, a, q) \mid q=0 \Rightarrow a_{2}=0\right\}$. Here the condition " $q=0 \Rightarrow a_{2}=0 "$ comes from Griffiths transversality, produces a slit and makes the space 
$J_{X / S, \Sigma_{0}}^{2}$ Hausdorff for the usual topology and hence for our topology of $J_{X / S, \Sigma_{0}}^{2}$ (defined by using the strong topology in [KU99, KU09, 3.1]) which is stronger than the usual topology.

\section{Comments on relative completeness.} We have an embedding

$$
\operatorname{Mor}\left(?, J_{X / S, \Sigma}^{r}\right) \subset \mathcal{E} x t_{L M H S}^{1}\left(\mathbf{Z}, H^{2 r-1}(X / S)(r)\right)
$$

of functors from the category ( $\mathrm{fs} / S$ ) of fs log analytic spaces over $S$ to the category of sets. Here, $\mathcal{E} x t^{1}$ is taken in the category (LMHS) of log mixed Hodge structures with polarized graded quotients (see [KU09] 2.6, [KKN08a] 2.3, 2.5 for the definition of log mixed Hodge structures).

Proposition. Let $\Sigma$ be a relatively complete fan (§2). Then, for any fs log analytic space $S^{\prime}$ over $S$ and any $a \in \operatorname{Ext}_{S^{\prime}}^{1}\left(\mathbf{Z}, H^{2 r-1}(X / S)(r)\right)$, locally on $S^{\prime}$, there is a log modification $S^{\prime \prime} \rightarrow S^{\prime}$ ([KU09] 3.6) and a subdivision $\Sigma^{\prime}$ of $\Sigma$ satisfying (1) in Theorem in $\S 2$ such that the image of a in $\operatorname{Ext}_{S^{\prime \prime}}^{1}\left(\mathbf{Z}, H^{2 r-1}(X / S)\right.$ $(r))$ belongs to $\operatorname{Mor}\left(S^{\prime \prime}, J_{X / S, \Sigma^{\prime}}^{r}\right)$.

The proof of the above fact is similar to the pure case [KU09] 4.3, where the extensions of period maps are explained.

In particular, we have the following For any fs $\log$ analytic space $S^{\prime}$ over $S$ which is $\log$ smooth over C ([KU09] 2.1.11), let $U$ be the open subspace of $S^{\prime}$ where the $\log$ structure is trivial. Let $a \in$ $\operatorname{Ext}_{U}^{1}\left(\mathbf{Z}, H^{2 r-1}(X / S)(r)\right)$ be an extension of graded polarized variation of MHS, regarded as a morphism $a: U \rightarrow J_{X_{U} / U}^{r}$ to the usual intermediate Jacobian. Assume that $a$ is admissible with respect to $S^{\prime}$. Then, locally on $S^{\prime}$, there is a $\log$ modification $S^{\prime \prime} \rightarrow S^{\prime}$ with $U \subset S^{\prime \prime}$ and a subdivision $\Sigma^{\prime}$ of $\Sigma$ satisfying (1) in Theorem such that $U \stackrel{a}{\rightarrow} J_{X_{U} / U}^{r} \rightarrow$ $J_{X / S, \Sigma^{\prime}}^{r}$ extends to a morphism $S^{\prime \prime} \rightarrow J_{X / S, \Sigma^{\prime}}^{r}$.

More specifically, assume $S^{\prime}=S=\Delta$. Then, $\operatorname{Ext}_{S^{\prime}}^{1}\left(\mathbf{Z}, H^{2 r-1}(X / S)(r)\right)$ is nothing but the space of admissible normal functions ([Sai96]), and the above fact says that any admissible normal function extends to some log intermediate Jacobian because, in this case, there is no non-trivial log modification, that is, $S^{\prime \prime}=S^{\prime}$.

Since a cycle on $X$ gives an admissible normal function by a theory of Saito ([Sai90, Sai96]), we also have the Abel-Jacobi map into the log intermediate Jacobian.

8. Remarks on Néron models. Here the base $S$ is any fs $\log$ analytic space unless otherwise stated. Let $H^{\prime}$ be a polarized log Hodge structure of weight -1 over $S$.

8.1. (The case where $F^{1}=0$ of this 8.1 is in [KKN08b] §5.)

From the viewpoint of the theory of log intermediate Jacobian, it is fundamental to consider the exact sequences $0 \rightarrow H^{\prime} \rightarrow\left(\mathcal{O}^{\log } \otimes H^{\prime}\right) / F^{0} \rightarrow H^{\prime} \backslash$ $\left(\mathcal{O}^{\log } \otimes H^{\prime}\right) / F^{0} \rightarrow 0$ of abelian sheaves on $\left(\mathrm{fs}_{\mathrm{s}} / S\right)^{\log }$ (see $\left[\right.$ KKN08a] for the definition of $(\mathrm{fs} / S)^{\log }$ ) and the induced

$$
\begin{aligned}
0 & \rightarrow \tau_{*} H^{\prime} \rightarrow\left(H_{\mathcal{O}}^{\prime} / F^{0}\right)^{\text {hor }} \\
& \rightarrow\left(\tau_{*}\left(H^{\prime} \backslash\left(\mathcal{O}^{\log } \otimes H^{\prime}\right) / F^{0}\right)\right)^{\text {hor }} \stackrel{\partial}{\rightarrow} R^{1} \tau_{*} H^{\prime}
\end{aligned}
$$

of abelian sheaves on (fs/S), where "hor" means the horizontal parts, i.e., the parts consisting of sections corresponding to pre-log mixed Hodge structures that satisfy the small Griffiths transversality. (Here the small Griffiths transversality is a weaker version of Griffiths transversality which appears in the definition of log mixed Hodge structure in [KU09] 2.6 , and which is imposed only at the points of degeneration.)

There are several important subgroups of $\left(\tau_{*}\left(H^{\prime} \backslash\left(\mathcal{O}^{\log } \otimes H^{\prime}\right) / F^{0}\right)\right)^{\text {hor }}$ which are, respectively, the inverse images of some subgroups of the monodromy group $R^{1} \tau_{*} H^{\prime}$ under the connecting homomorphism $\partial$ in the last exact sequence $(*)$. The sheaf $\mathcal{E} x t_{L M H S}^{1}\left(\mathbf{Z}, H^{\prime}\right)$ is one of them, which is the inverse image of the "admissible part" of the monodromy group. From the viewpoint of log geometry, it is this sheaf that should be called the "log intermediate Jacobian", and what have been called log intermediate Jacobians so far in this article should be called "models of the log intermediate Jacobian."

Note that this sheaf $\mathcal{E} x t_{L M H S}^{1}\left(\mathbf{Z}, H^{\prime}\right)$ is a group object, and, in a sense, log smooth (even when $H^{\prime}$ degenerates), as so are log complex tori introduced in [KKN08a]. The authors expect that it would be possible to generalize the theory of log complex tori and the theory of their proper models developed in [KKN08a] and [KKN08b] to the log intermediate Jacobians.

8.2. Let the situation be as in $\S \S 0-1$. Let $\Sigma_{1}$ be the fan consisting of $\{0\}$ and the cones $\left(\mathbf{R}_{\geq 0}\right) N$ for $N \in \mathfrak{g}_{\mathbf{Q}}$ satisfying $\left.N\right|_{H^{\prime}}=N^{\prime}, N(e)=N^{\prime}(a)$ for some $a \in H_{\mathbf{Q}}^{\prime}$ such that $\gamma a-a \in H^{\prime}$. We define the Néron model as $J_{X / S, \Sigma_{1}}^{r}$. This is a log manifold whose $\log$ structure is the inverse image of that of the base, 
and "represents" (in some suitable senses) the subgroup of $\left(\tau_{*}\left(H^{\prime} \backslash\left(\mathcal{O}^{\log } \otimes H^{\prime}\right) / F^{0}\right)\right)^{\text {hor }}$ which is the inverse image of $\iota^{-1}\left(\left(R^{1} \tau_{S *} H^{\prime}\right)_{\text {tor }}\right) \subset R^{1} \tau_{*} H^{\prime}$ by $\partial$ in $(*)$. Here $\tau_{S}: S^{\log } \rightarrow S$ and $\iota:(\mathrm{fs} / S) \rightarrow S$ are the natural morphisms. Precisely, we can say as follows: In general, $J_{X / S, \Sigma}^{r}$ is defined as an object of $\mathcal{B}(\log )$ ([KU09] 3.2.4) (even if $S$ is not $\Delta$ but any fs log analytic space). If we do 8.1 over $\mathcal{B}(\log ) / S$ instead of (fs/S), we can say $J_{X / S, \Sigma_{1}}^{r}$ represents the indicated subgroup in the usual sense. Alternatively, in general, if the base $S$ is $\log$ smooth ([KU09] 2.1.11), $J_{X / S, \Sigma}^{r}$ is a $\log$ manifold, and any $\log$ manifold is characterized by its induced sheaf on (fs $/ S$ ). Note that this $J_{X / S, \Sigma_{1}}^{r}$ generalizes Clemens' model which is constructed in $\S 5$ under the condition (5.1).

It is easy to see that for a sufficiently large $L$ and for some base of $Q \cap L$ (and for any $s$ ), our fan $\Sigma$ in $\S 3$ contains $\Sigma_{1}$ as a subfan. Hence our log intermediate Jacobian associated to $\Sigma$ contains the Néron model as an open subspace. In this sense, our construction gives a kind of compactification of the Néron model. See $\S 5$ for the special case of this fact under the condition (5.1).

By the proof of [KU09] 4.3.1 (i), which works also in this mixed Hodge theoretic situation, any admissible normal function extends to the Néron model.

The relationship does not seem to be known between this $J_{X / S, \Sigma_{1}}^{r}$ and the Néron model constructed by Green-Griffiths-Kerr [GGK.p].

\section{References}

[ Car80 ] J. A. Carlson, Extensions of mixed Hodge structures, in Journées de Géometrie Algébrique d'Angers, Juillet 1979/Algebraic Geometry, Angers, 1979 (A. Beauville, eds.), 107-127, Sijthoff \& Noordhoff, Alphen aan den Rijn, 1980.

[ CKS86 ] E. Cattani, A. Kaplan and W. Schmid, Degeneration of Hodge structures, Ann. of Math. (2) 123 (1986), no. 3, 457-535.

[ Cle83 ] H. Clemens, The Néron model for families of intermediate Jacobians acquiring "algebraic" singularities, Inst. Hautes Études Sci. Publ. Math. 58 (1983), 5-18.

[ EZ84 ] F. El Zein and S. Zucker, Extendability of normal functions associated to algebraic cycles, in Topics in transcendental algebraic geometry (Princeton, N.J., 1981/1982), Ann. of Math. Stud., 106, Princeton Univ. Press, Princeton, NJ, 1984, 269-288.

[ Fuj99 ] T. Fujisawa, Limits of Hodge structures in several variables, Compositio Math. 115 (1999), no. 2, 129-183.
[ GGK.p ] M. Green, P. Griffiths and M. Kerr, Néron models and limits of Abel-Jacobi mappings. (Preprint).

Gri68 ] P. A. Griffiths, Periods of integrals on algebraic manifolds. I. Construction and properties of the modular varieties, Amer. J. Math. 90 (1968), 568-626.

Gri70 ] P. A. Griffiths, Periods of integrals on algebraic manifolds: Summary of main results and discussion of open problems, Bull. Amer. Math. Soc. 76 (1970), 228-296.

GS69 ] P. A. Griffiths and W. Schmid, Locally homogeneous complex manifolds, Acta Math. 123 (1969), 253-302.

Ill94 ] L. Illusie, Logarithmic spaces (according to K. Kato), in Barsotti Symposium in Algebraic Geometry (Abano Terme, 1991) (V. Cristante and $W$. Messing, eds.), Perspectives in Math. 15, Academic Press, San Diego, CA, 1994, 183-203.

[KKN08a] T. Kajiwara, K. Kato and C. Nakayama, Logarithmic abelian varieties, Part I: Complex analytic theory, J. Math. Sci. Univ. Tokyo 15 (2008), no. 1, 69-193.

[KKN08b] T. Kajiwara, K. Kato and C. Nakayama, Analytic $\log$ Picard varieties, Nagoya Math. J. 191 (2008), 149-180.

[ Kas85 ] M. Kashiwara, The asymptotic behavior of a variation of polarized Hodge structure, Publ. Res. Inst. Math. Sci. 21 (1985), no. 4, 853-875.

Kas86 ] M. Kashiwara, A study of variation of mixed Hodge structure, Publ. Res. Inst. Math. Sci. 22 (1986), no. 5, 991-1024.

Kaf98 ] F. Kato, The relative log Poincaré lemma and relative log de Rham theory, Duke Math. J. 93 (1998), no. 1, 179-206.

Kak89 ] K. Kato, Logarithmic structures of FontaineIllusie, in Algebraic analysis, geometry, and number theory (J.-I. Igusa, ed.), (Baltimore, $M D, 1988)$, Johns Hopkins Univ. Press, Baltimore, MD, 1989, 191-224.

[KMN02 ] K. Kato, T. Matsubara and C. Nakayama, $\log C^{\infty}$-functions and degenerations of Hodge structures, in Algebraic geometry 2000, Azumino (Hotaka), Adv. Stud. Pure Math., 36, Math. Soc. Japan, Tokyo, 2002, 269-320.

KN99 ] K. Kato and C. Nakayama, Log Betti cohomology, log étale cohomology, and log de Rham cohomology of log schemes over C, Kodai Math. J. 22 (1999), no. 2, 161-186.

[ KNU08 ] K. Kato, C. Nakayama and S. Usui, SL(2)orbit theorem for degeneration of mixed Hodge structure, J. Algebraic Geom. 17 (2008), no. 3, 401-479.

[ KNU09 ] K. Kato, C. Nakayama and S. Usui, Classifying spaces of degenerating mixed Hodge structures, I: Borel-Serre spaces, in Algebraic analysis and around, Adv. Stud. Pure Math., 54, Math. Soc. Japan, Tokyo, 2009, $187-222$. 
[ KNU.p ] K. Kato, C. Nakayama and S. Usui, Classifying spaces of degenerating mixed Hodge structures, III: spaces of nilpotent orbits. (in preparation).

[ KU99 ] K. Kato and S. Usui, Logarithmic Hodge structures and classifying spaces, in The arithmetic and geometry of algebraic cycles (Banff, AB, 1998), CRM Proc. \& Lect. Notes 24, Amer. Math. Soc., Providence, RI, 1999, 115-130.

[ KU09 ] K. Kato and S. Usui, Classifying spaces of degenerating polarized Hodge structures, Ann. of Math. Stud., 169, Princeton Univ. Press, Princeton, NJ, 2009.

[ Mat98 ] T. Matsubara, On log Hodge structures of higher direct images, Kodai Math. J. 21 (1998), no. 2, 81-101.

[ Sai90 ] M. Saito, Mixed Hodge modules, Publ. Res. Inst. Math. Sci. 26 (1990), no. 2, 221-333.

[ Sai96 ] M. Saito, Admissible normal functions, J. Algebraic Geom. 5 (1996), no. 2, 235-276.
[ Sch73 ] W. Schmid, Variation of Hodge structure: The singularities of the period mapping, Invent. Math. 22 (1973), no. 3-4, 211-319.

Ste76 ] J. H. M. Steenbrink, Limits of Hodge structures, Invent. Math. 31 (1975/76), no. 3, 229-257.

SZ85 J J. H. M. Steenbrink and S. Zucker, Variation of mixed Hodge structure. I, Invent. Math. 80 (1985), no. 3, 489-542.

[ Usu84 ] S. Usui, Variation of mixed Hodge structure arising from family of logarithmic deformations II: Classifying space, Duke Math. J. 51 (1984), no. 4, 851-875.

[ Usu01 ] S. Usui, Recovery of vanishing cycles by log geometry, Tohoku Math. J. (2) 53 (2001), no. $1,1-36$.

Zuc76 ] S. Zucker, Generalized intermediate Jacobians and the theorem on normal functions, Invent. Math. 33 (1976), no. 3, 185-222. 